\title{
Review
}

\section{An Overview of the Effects of Light on Human Circadian Rhythms: Implications for New Light Sources and Lighting Systems Design}

\author{
Mariana G. FIGUEIRO \\ Lighting Research Center, Rensselaer Polytechnic Institute, USA
}

Received May 2, 2013, Accepted July 25, 2013

This paper is based on the authors' presentation given at the 13th International Symposium on Science and Technology of Lighting (LS-13) held June 24-29, 2012, in Troy, New York, USA.

\begin{abstract}
Circadian rhythms are seen at every level of biology, from single cells to complex behaviors. The timing of every biological function in mammals is governed by the master clock in the suprachiasmatic nuclei (SCN), which has an intrinsic period of slightly longer than 24 hours. The light/dark pattern incident on the retina synchronizes the SCN to the 24-hour local time, coordinating and enabling diverse biological functions to occur at the correct time of day and night for optimum species survival. Without exposure to a regular, daily pattern of light and dark, circadian rhythms become disrupted. A wide range of modern maladies, from sleep disorders to cancer, has been linked to light-induced circadian disruption. Light has, however, been defined only in terms of the human visual system, not the circadian system. Light source and systems development should consider the needs of both the visual and non-visual systems. Discussed are the lighting characteristics impacting these two systems and the implications for designing light for various healthcare and medical applications.
\end{abstract}

KEYWORDS: circadian, melatonin, non-visual effects of light, older adults sleep, teenagers sleep

\section{Introduction}

Light is not just for vision. Light can affect human behavior, mood and health via pathways other than the visual system. One of the important pathways via which light can affect humans is through the circadian system. While knowledge in this area is still being developed, much is already known and a summary of current research is presented. The present paper also discusses practical lighting solutions that are based on fundamental research on the effects of light on circadian rhythms. It is hoped that the information presented in this paper will motivate designers, manufacturers, and users of light to apply lighting differently in their environments, so that the lives of those suffering from circadian sleep disorders, such as older adults, including those with Alzheimer's disease (AD), teenagers, and shift workers can be improved.

\section{Circadian rhythms}

Because the Earth rotates on its axis, there is a regular and predictable 24-hour pattern of daylight and darkness over most of its surface. Terrestrial species have adapted to this daily pattern by evolving biological rhythms that repeat at approximately 24 -hour intervals. These rhythms are called circadian rhythms, from the Latin circa (about) and dies (day), and reflect a very tight coupling between the natural environmental light/ dark pattern and the cyclicity of the endogenous master clock, located in the suprachiasmatic nuclei (SCN). The master clock governs a wide range of biological cycles, from cell division, to hormone production, to behavior that, when synchronized with the natural light/dark cycle, enables the organism to entrain these cycles to its particular photic niche (diurnal or nocturnal) and to its location on Earth. Although the period of the solar cycle is almost exactly 24 hours, the period of the internal, genetically self-governing master clock is slightly longer than 24 hours in most diurnal species and slightly shorter in most nocturnal species.

Environmental cues can reset and synchronize the SCN daily, ensuring that humans' behavioral and physiological rhythms are in synchrony with the daily rhythms found within their environment. Although the circadian system shares receptors and neurons in the retina with the visual system, the retinal ganglion cells exiting the eye for the visual centers are different from those exiting the eye for the circadian system. In 2002, the intrinsically photosensitive retinal ganglion cell (ipRGC), a novel photoreceptor type in the retina, was discovered ${ }^{1)}$. The ipRGCs are central to an important "non-visual" response to light by the retina, most notably the regulation of circadian rhythms. Although the 
ipRGCs are the primary photoreceptors for the circadian system, it is now well accepted that classical photoreceptors in the retina (rods and cones) also participate in how the retina converts light signals into neural signals for the circadian system.

The most commonly measured master clock output rhythms include hormone production, core body temperature, sleep/wake cycles, and rest/activity rhythms. Melatonin is a hormone produced by the pineal gland at night and under conditions of darkness. Because melatonin production is associated with nighttime, it is called the hormone of darkness. Melatonin gives the body time cues, and is used as a marker of the circadian clock. It can be measured in plasma, saliva, or urine. Sufficient light at night will acutely cease melatonin production and, depending on the timing of exposure, light can phase shift the timing of melatonin production. Core body temperature is also used as a marker of the circadian clock, and has an inverse relationship with melatonin. It reaches a peak late in the afternoon/early evening and a trough late at night/early in the morning. The sleep/wake cycle and rest/activity pattern of an entrained person are also output rhythms and can be measured in laboratory conditions or in the field.

\section{Lighting characteristics affecting the human circadian system}

Important lighting characteristics affecting the human circadian system include quantity, spectral power distribution of the light source, timing and duration of exposure, spatial distribution, and light history. The circadian system responds to light differently than the visual system ${ }^{2}$. In terms of quantity, recommended light levels for the built environment are solely based on the needs of on the visual system. The quantity of polychromatic "white" light necessary to activate the circadian system is at least two orders of magnitude greater than the amount that activates the visual system. In terms of spectrum, the circadian system is maximally sensitive to short-wavelength ("blue") light, with a peak spectral sensitivity at around $460 \mathrm{~nm}^{3)-5 \text { ), }}$ while the visual system is most sensitive to the middlewavelength portion of the visible spectrum at around $555 \mathrm{~nm}$. In terms of temporal characteristics, operation of the visual system does not depend significantly on the timing of light exposure, and thus responds well to a light stimulus at any time of the day or night. On the other hand, the circadian system is dependent on the timing of light exposure; light can phase advance or phase delay the master $\operatorname{clock}^{617}$. In addition, while the visual system responds to a light stimulus very quickly (less than one second), the duration of light exposure needed to affect the circadian system can take minutes $^{2}$. For the visual system, spatial light distribution is critical (e.g., when reading black letters on white paper), while the circadian system does not respond to spatial patterns. One study showed that light reaching the lower retina is more effective in suppressing melatonin than light reaching the upper retina ${ }^{8}$, but it is not yet well-established how light incident on different portions of the retina will affect the circadian system. It is also important to note that the short-term history of light exposure affects the sensitivity of the circadian system to light; the higher the exposure to light during the day, the lower the sensitivity of the circadian system to light at night, as measured by nocturnal melatonin suppression $^{9)}$ and phase shifting ${ }^{10)}$.

\section{Light and health applications}

The therapeutic value of circadian light (and dark) on fringe populations has been demonstrated in laboratory and field studies. Light of the appropriate characteristics can reduce symptoms of seasonal affective disorder ${ }^{11)}$, increase sleep efficiency of older adults ${ }^{12}$, including those with Alzheimer's disease and related dementia $(\mathrm{ADRD})^{13) 144}$, improve circadian entrainment of premature infants ${ }^{15}$, help teenagers wake up earlier in the morning ${ }^{16)}$, and increase alertness and well-being of nightshift workers ${ }^{17}$. It has even been suggested that some forms of cancer may be linked to the disruption of the normal circadian light/dark pattern ${ }^{18}$. . The impact of light on the general population outside laboratory conditions is less clear. Discussed here are a few of the light and health applications.

\subsection{Older adults and Alzheimer's disease patients}

Sleep disturbances in older adults are quite common. Seniors living in assisted living facilities are perhaps the best example of a population at risk of circadian disorders that could be alleviated by light treatment. Due to age-dependent reduced retinal light exposures and to fixed lighting conditions in their living environments, possibly compounded by loss of neurons, a less sensitive circadian clock, and diminished communication between the SCN and the downstream rhythms, seniors are less likely to experience the necessary, robust, 24-hour light/dark pattern needed for circadian entrainment.

Bright white light (2500 lux at the cornea) can help reduce the negative impact of aging on circadian rhythms of sleep and wake, and thus improve the quality and quantity of sleep in older adults, including those with ADRD. Fetveit et al. ${ }^{19)}$ demonstrated that exposure to 2 hours of bright light in the morning for at least 2 weeks substantially improved the sleep efficiency of older adults living in nursing homes. Alessi et al. ${ }^{20)}$ showed that five consecutive days of 30-minute exposure to sunlight, increased physical activity, structured bedtime, and control of light and noise at night resulted in a significant decrease in daytime sleeping in intervention participants compared to controls. Further- 
more, they showed that intervention participants had increased participation in social and physical activities, as well as social conversation. Murphy and Campbell ${ }^{21)}$ have shown that light exposure in the evening can delay the circadian clock and help older adults sleep better at night and be more awake during the day.

Analyzing actigraph data, Van Someren et al. ${ }^{14)}$ found that increased illumination ( $\geq 1300$ lux) in the living environments of ADRD patients increased the stability of their rest/activity rhythm following four weeks of treatment. Interdaily stability (IS), which is the strength of coupling of the rhythm to the supposed stable environmental zeitgebers, increased after treatment. The intradaily variability (IV), which gives an indication of the fragmentation of the rhythm (i.e., the frequency and extent of transitions between rest and activity), decreased after treatment. In ADRD patients, Satlin et $\mathrm{al}^{22)}$ found an increase in circadian amplitude and improvement in IV, but not in IS. They also found that evening light exposure (1500-2000 lux, 7-9 p.m.) decreased nighttime activity and sundowning symptoms in the subjects with ADRD. Lyketsos et al. ${ }^{23)}$ administered morning light (10,000 lux) for two weeks to institutionalized ADRD patients and found that those who exhibited agitated behaviors slept more hours at night with the bright light exposure. Ancoli-Israel et al. ${ }^{24)}$ administered 2500 lux of light for two hours in the morning or evening for ten consecutive days and found both groups to have more consolidated sleep at night. Yamadera et al. $^{25}$ ) found that morning bright light administration (3000 lux, 9-11 a.m. for four weeks) resulted in improved mental status scores, decreased percentage of daytime naps/naptime, increased percentage of nighttime sleep time, and decreased percentage of nighttime awakenings.

Dowling et al. ${ }^{26)}$ tested the effects of morning versus evening light, hypothesizing that morning (9:3010:30 a.m.) bright light ( $\geq 2500$ lux) should result in the most improvement, with a phase advance in the rest/ activity rhythm. Conversely, afternoon light exposure (from 3:30-4:30 p.m.) would phase delay the rhythm. No significant differences in actigraphy-based measures of nighttime sleep or daytime wake were found. However, both groups evidenced a significantly more stable rest/ activity rhythm acrophase over the ten-week treatment period compared to the controls. The study concluded that one hour of bright light exposure may provide sufficient additional input to the circadian pacemaker to facilitate entrainment to the 24-hour day. When examining morning light therapy treatment, their results indicated that subjects with the most impaired rest/activity rhythms responded significantly and positively to a one-hour light intervention. Sloane and colleagues ${ }^{27}$ showed a statistically significant improvement in nighttime sleep with morning or all-day light ( $\geq 2500$ lux), with greater improvement among persons with severe dementia. The authors claimed that the effect size was greater than has been reported using prescription sleep medicines in long-term care populations.

Although widely known that the circadian system is maximally sensitive to short-wavelength ("blue") light, only four studies to date, however, have investigated the effects of short-wavelength light [either narrowband 470-nm light or high correlated color temperature (CCT) white light] on sleep patterns in older adults, including those with ADRD. In 2002, we showed that evening exposure to 30 lux at the cornea of 470-nm light from light emitting diodes (LEDs) for two hours consolidated rest/activity rhythms and increased sleep efficiency of older people with and without ADRD ${ }^{1213)}$. A study published in 2011, using a very similar protocol as the one we used in 2002, also showed a beneficial effect of narrowband, short-wavelength light on sleep efficiency in older adults without dementia ${ }^{28}$. In 2008 , we showed that 400 lux of a $6500 \mathrm{~K}$ light delivered at the cornea of healthy older adults with sleep disturbances increased their light/dark ratio, as measured via actigraphy ${ }^{29}$.

To determine whether the progression of cognitive and non-cognitive symptoms may be ameliorated by individual or combined long-term application of bright light and melatonin, Riemersma-van der Lek and colleagues ${ }^{30)}$ conducted a long-term, double-blind, placebocontrolled study with 189 older adults living in nursing homes. Light alone was found to attenuate both cognitive deterioration by as much as $5 \%$, as determined by the Mini Mental Status Examination, and the increase in functional limitations, as determined by the Activities of Daily Living Scale. Oral melatonin alone shortened sleep onset latency and increased sleep duration, but also increased withdrawn behavior. The light and melatonin treatment increased sleep efficiency and improved nocturnal restlessness, thus indicating that the adverse effect of melatonin on mood can be counteracted when administered as part of treatment with light for cognitive and non-cognitive function.

Using the Dimesimeter, a calibrated light meter developed by the Lighting Research Center ${ }^{31}$, we have been able to characterize the light/dark and activity/rest patterns in persons with ADRD and in healthy older adults. Persons with ADRD experienced lower light levels, exhibited lower activity levels, and had greater levels of circadian disruption than healthy older adults during winter. Seasonal differences were observed for persons with ADRD; lower levels of light exposure and greater levels of circadian disruption, as measured by phasor analyses, were seen during the winter than during the summer, although activity levels did not differ for the two seasons. These ecological data suggest that light exposures in those with ADRD can be the underlying cause of the sleep disturbances so commonly found in 
this population, especially in winter months. Therefore, light sources delivering higher circadian stimulation during the daytime hours have the potential to improve sleep in this population, especially in winter months. Practical and effective light delivery methods, however, should be made available to homeowners, facility managers, and designers.

\subsection{Teenagers}

Teenagers tend to go to sleep late and wake up late (sometimes three to six hours later than normal sleeping hours). This pattern interferes with their normal functioning because teenagers have difficulty waking up in the morning for school. Because they typically fall asleep late, they do not sleep for as many hours as those going to sleep at more normal hours. Delayed sleep patterns commonly experienced by adolescents are probably associated with hormonal changes that occur at puberty. Bright light exposure ( $\geq 2500$ lumens) after minimum core body temperature and dim light exposure during the evening have been shown to advance the phase of the master clocks of delayed persons ${ }^{32}$. Some evidence has emerged suggesting that the endogenous circadian period is longer ${ }^{33} 34$ ), and the sensitivity to evening light is greater in adolescents than in adults, especially for those suffering from delayed sleep phase disorder ${ }^{35}$. Both of these phenomena would functionally delay the timing of the adolescent circadian clock, thereby contributing to later bed and rise times. Moreover, it has been suggested that adolescents accumulate sleep pressure more slowly over the course of the day ${ }^{36)}$, further contributing to a later sleep onset ${ }^{37}$.

Exposure to irregular light/dark patterns may have a particularly high impact on teenagers. Rigid school schedules require teens to be in class early in the morning, yet schools may not provide adequate light or daylight to stimulate their circadian system, especially in dark winter months. Since the circadian system responds to short-wavelength light, the electric light in classrooms may be insufficient for stimulating the brain's clock, even though it is perfectly adequate for reading. As teenagers spend more time indoors, they may miss out on essential morning light needed to stimulate their circadian systems and promote entrainment to the 24-hour solar day. In late spring, if teenagers spend more time outdoors after school, the brain's clock may be delayed by the evening daylight exposure. Late spring mornings may also pose a concern because of the very early sunrises. Students who are phase delayed, and thus reach their minimum core body temperature at later clock hours in the morning, may run the risk of receiving too much daylight in their phase delay portion of the phase response curve while traveling to school in the early morning.

Using this information, we conducted two field stud- ies $^{38-40)}$ to investigate the impact of light exposures on dim light melatonin onset (DLMO), a primary marker for the timing of the master clock, and on sleep duration for two populations of eighth graders. It was hypothesized for one study conducted in North Carolina that the lack of short-wavelength light in the morning would delay the timing of the students' DLMOs. For the other study conducted in New York, it was hypothesized that exposure to more evening light in spring relative to winter would also delay the DLMOs of adolescents. In all studies, the student subjects wore the Daysimeter ${ }^{41}$, which measured actual circadian light exposures for the study weeks.

The study conducted in North Carolina was divided in two phases. In the first phase, we examined how restriction of short-wavelength, circadian light exposure impacted evening DLMO $^{38}$. This field study was conducted at a middle school with unusually high levels of daylight in the classrooms. When 11 teenage subjects wore special orange glasses in the morning to remove short-wavelength, circadian light (Figure 1) for five consecutive school days, their DLMO was delayed by about 30 minutes compared to the previous week, when they did not wear the glasses (Figure 2). The orange glasses allowed them to see well enough to perform their visual tasks, but their circadian pacemaker was not receiving enough stimulation in the morning.

In the second phase of this study, 22 students participated in a 5-day field study conducted at the same middle school ${ }^{40)}$. Half of the students studied wore the orange glasses that removed short-wavelength light for five consecutive days, while a control group $(n=11)$ did not wear them. DLMO was significantly delayed (approximately 30 minutes) for those students who wore orange glasses compared to the control group. Sleep durations were slightly, but not significantly,

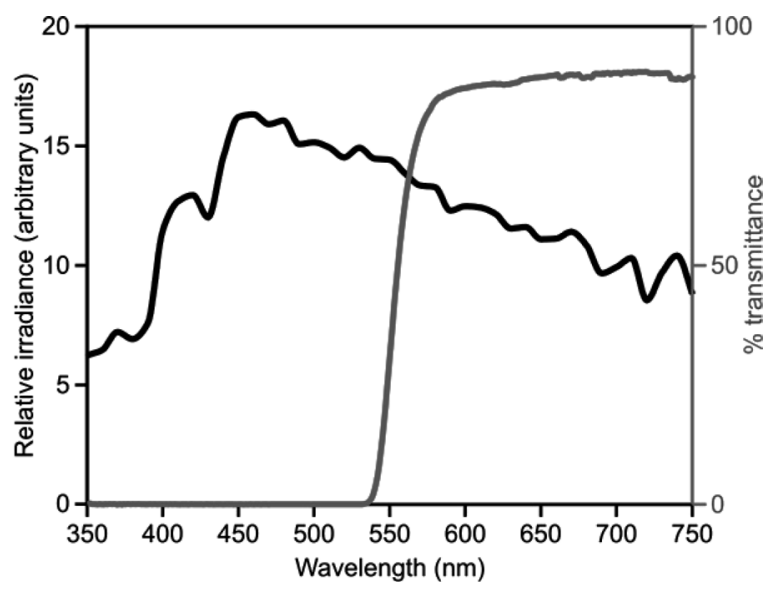

Figure 1 The spectral irradiance distribution of a phase of daylight $(6500 \mathrm{~K})$ and the spectral transmittance of the orange glasses. Short wavelengths below approximately $540 \mathrm{~nm}$ are removed ${ }^{38)}$. 


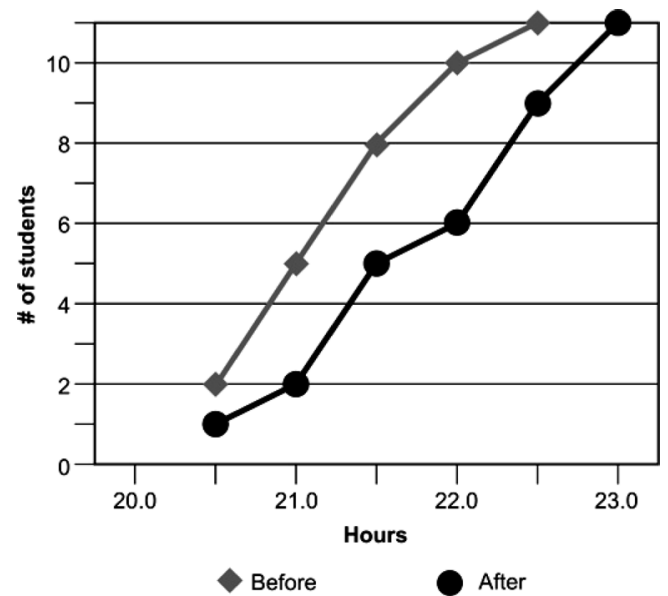

Figure 2 Cumulative frequencies of DLMO times before and after students wore the orange glasses in phase 1 of the study.

curtailed in the orange-glasses group. There was no significant difference in the performance of short-term reaction times and memory tests between the two groups; however, morning circadian light was removed for 5 days only and it is not known whether chronic deprivation of morning short-wavelength light will reduce performance.

In the second study, 16 teenage students participated in a seasonal study in upstate New York. Evening melatonin onset was measured one winter evening and one spring evening in the same subjects. DLMO was delayed by about 20 minutes in the spring relative to that in winter (Figure 3). Sleep onset was also significantly delayed in the spring, by about 16 minutes relative to that in the winter ${ }^{39}$. Evening light exposures were significantly greater in spring than in winter, which could explain why DLMO in the same group of students was delayed in spring months compared to winter months.

The delays in DLMO and sleep onset shown in the studies were consistent with the measured differential circadian light exposures. Namely, restricting circadian light exposure in the morning and extending it in the evening delayed evening DLMO and sleep onset.

Given these facts, to help minimize sleep restriction and possibly circadian disruption, teenagers should receive higher levels of morning light (or daylight) in schools and lower levels of evening light (or daylight) at home. The results from the field studies demonstrate, through real-life applications, the well-founded concepts of circadian physiology and human response to light from laboratory studies. More importantly, the present studies demonstrate that light treatment for correcting circadian phase will only be successful if the overall 24hour light/dark exposure is measured and controlled.

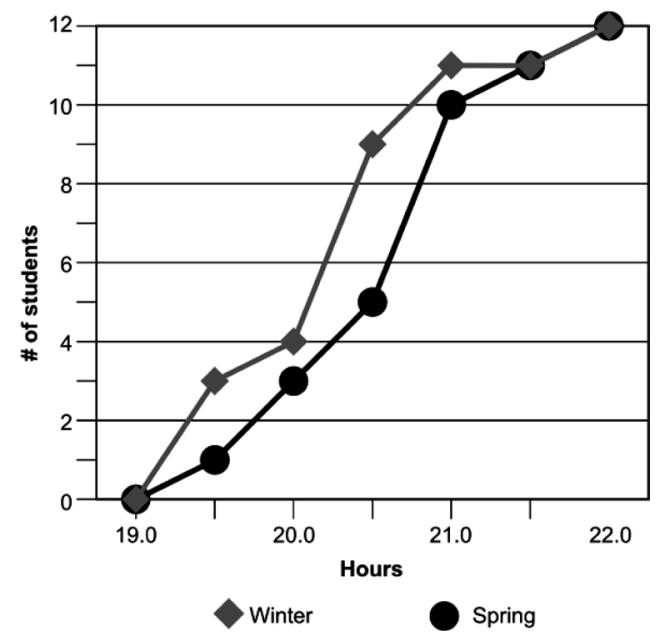

Figure 3 Cumulative frequencies showing the number of students who reached DLMO between 19.0 and 22.0 hours. DLMO was delayed by about 20 minutes in the spring relative to the winter.

\subsection{Graveyard shift}

Rotating-shift nurses workers are typically entrained to a dayshift schedule. Therefore, coping with nightshift becomes harder as a result of the natural tendency to be asleep at night. Light can acutely impact alertness in rotating-shift workers or it can phase shift the timing of their master clocks. Acute effects are much easier to achieve because their benefits are perceived shortly after the exposures. Phase shifting effects, on the other hand, allow shift workers to cope with being awake at night by providing entrainment to the nightshift. Light exposure control throughout the 24 hours is, however, needed in order to maintain entrainment to the nightshift, making it harder for workers to comply with the new light regimen. The reversed schedule would only work for permanent-shift workers, rather than rotatingshift workers who work 2-4 nights per week.

Studies have shown that acute exposure to high levels of bright light at night (levels typically $>2500$ lux at the cornea) increases alertness, measured subjectively and objectively. Badia and colleagues ${ }^{42)}$ showed that exposure to 90 minutes of a 5000 to 10,000 lux of white light during the nighttime increased brain activities in the alerting range (beta range). Cajochen and colleagues ${ }^{43)}$ also showed that exposure to 3190 lux at the eye of white light increased brain activities, and that much lower levels of short-wavelength light increased brain activities and reduced sleepiness at night ${ }^{44)}$. In a field study, we showed that 15-minute exposure to 2500 lux on the workplane delivered via metal halide table lamps improved subjective ratings and increased tympanic temperatures of nurses working in a Newborn Intensive Care Unit ${ }^{17}$.

Phase shifting the circadian system using light has 
been shown to be an effective way to increase adaptation to the nightshift because the worker is no longer producing melatonin at night; rather, as a result of a 12-hour phase shift, workers produce melatonin during the day, when they are supposed to be asleep. This is certainly the preferred lighting solution because it minimizes any adverse effects of being awake while the circadian clock is ready to go to sleep. However, lifestyle changes are required in order to accomplish and maintain the shift in the timing of the circadian clock because light exposure needs to be controlled throughout the 24 hours. Once entrained to the nightshift schedule, workers would have to increase the amount of circadian light they are exposed to while at work (i.e., equivalent to their daytime light exposure) and protect themselves from daytime light, especially the morning light they receive during their commute home. This can be accomplished with personal light treatment devices, such as the use of blue-light goggles and use of orangetinted goggles to remove circadian light during the daytime hours. The safety of the orange-tinted glasses during the drive home is yet to be tested, however, but assuming that workers are entrained to the nightshift schedule, they should still be capable of driving home safely. It is also recommended that they have blackout shades in their bedrooms to mimic as much as possible the nighttime environment while they sleep. Improvement in certain types of performance tasks, greater subjective and objective alertness and wakefulness, better sleep quality during the day, and possibly better health are some of the benefits associated with circadian adaptation to nightshift work. Smith and colleagues ${ }^{45}$ proposed a compromise solution, where light is applied at the first half of the shift to delay the DLMO in rotating shift workers. In other words, they would become extreme "night owls" and their bedtimes would shift to later in the night, allowing them to cope better with staying awake at night and still be awake during the daytime hours when they were off their shift.

In most studies to date, the alerting effects of light have been linked to its ability to suppress melatonin ${ }^{46}$. In a recent study, however, we demonstrated that exposures to both short-wavelength (blue) and longwavelength (red) lights in the middle of the night increased beta and reduced alpha power relative to preceding dark conditions, although only blue light significantly suppressed melatonin relative to darkness ${ }^{47}$. Exposures to high (i.e., 40 lux at the cornea), but not low (i.e., 10 lux at the cornea), levels of red and of blue light significantly increased heart rate relative to darkness. We also demonstrated that both blue and red lights can significantly increase nighttime cortisol levels and performance, as measured by short-term reaction times and matching-to-sample tests ${ }^{48)}$. Our findings suggest that the melatonin pathway does not seem to be the only light-sensitive pathway that can affect alertness and performance at night. These results are consistent with studies showing alerting effects of light during the daytime, when melatonin levels are low ${ }^{49-51)}$.

The knowledge that long-wavelength light can increase alertness is important because, although shortwavelength light has been shown to increase alertness at night, it can also suppress melatonin. It has been hypothesized that the high incidence of breast cancer in industrialized society is, perhaps, caused by electricity in modern buildings through reductions in melatonin concentrations. Therefore, a light source that can increase alertness without suppressing melatonin may be an optimal solution for maintaining alertness in shift workers. Stevens ${ }^{18)}$ postulated that one of the electricity-induced reductions in melatonin was a result of electric light at night. The melatonin hypothesis stimulated various lines of research, from laboratory studies using animal models to epidemiological studies with humans. Suppression of melatonin was hypothesized to increase production of estrogens in the ovaries, which in turn would stimulate the turnover of breast epithelial stem cells, thereby increasing the likelihood of cancer $^{18)}$. Low melatonin levels in rats were also shown to increase cancer cell proliferation in existing tumors ${ }^{52)}$. Epidemiological studies showed that nightshift work, a surrogate for light at night exposure, increased the likelihood of breast and colorectal cancer ${ }^{53)}$. Thus, although no direct causal link has ever been established between breast cancer and light at night in architectural spaces, several researchers have suggested plausible links between increased cancer risk and electric light at night through melatonin depletion or disruption. Therefore, it is prudent to avoid suppressing melatonin in shift workers until this relationship is better understood. Although more research is recommended to establish the pathways in the brain associated with the alerting effects of light, using red, rather than blue, light at night might be a good option for increasing alertness without disturbing the melatonin rhythm in shift workers.

\section{Implications for light sources and lighting systems design}

As discussed above, light can have a profound impact on human health and well-being, but this relationship is not fully understood. It seems reasonable with the information available today, however, to design a 24-hour lighting scheme that maintains circadian entrainment as much as possible while promoting good visibility during waking hours and safe navigation at night. Following this logic, lighting in the built environment should provide a) high circadian light stimulation during the day and low circadian stimulation at night, b) good visual performance (e.g., reading) during waking hours, and c) low-level night-lights that enable safe movement 
Table 1 Predicted melatonin suppression by various light sources based on calculations using the Rea et al. model of human circadian phototransduction ${ }^{5) 55}$ (56).

\begin{tabular}{|c|c|c|}
\hline \multirow{2}{*}{ Light source } & \multicolumn{2}{|c|}{ Illuminance (lx) } \\
\hline & $25 \%$ suppression & $50 \%$ suppression \\
\hline Daylight (DIE D65) & 138 & 524 \\
\hline $2856 \mathrm{~K}$ incandescent A-lamp & 209 & 817 \\
\hline 2700 K CFL (Greenlite 15WELS-M) & 313 & 1223 \\
\hline $3350 \mathrm{~K}$ linear fluorescent (GE F32T8 SP35) & 302 & 1180 \\
\hline $4100 \mathrm{~K}$ linear fluorescent (GE F32T8 SP41) & 289 & 1069 \\
\hline 5200 K KED phosphor white (Luxeon Star) & 112 & 429 \\
\hline $6220 \mathrm{~K}$ linear fluorescent (Philips Colortone 75) & 144 & 549 \\
\hline 8000 K Lumilux Skywhite fluorescent (OSI) & 162 & 610 \\
\hline Blue LED (Luxeon Rebel, $\lambda_{\text {peak }}=470 \mathrm{~nm}$ ) & 13 & 52 \\
\hline
\end{tabular}

through the space and minimize sleep disruption ${ }^{5455)}$. Evidence suggests that high circadian stimulation during daytime waking hours can probably be achieved by about 600 lux at the cornea of a $6500 \mathrm{~K}$ light source. Table 1 shows the results of calculations using the model of human circadian phototransduction by Rea and colleagues $^{556)}$, which allows one to rank order the effectiveness of various light sources in suppressing nocturnal melatonin. The table shows calculated illuminance necessary to suppress melatonin by $25 \%$ and $50 \%$, given a 1-hour exposure and a pupil diameter of $2.3 \mathrm{~mm}$. Lower light levels may be used if longer exposure times are administered. As shown in Table 1, if a light source with a higher CCT is used, the circadian stimulation will be no more than twice the effectiveness of a warmer light source, so using a higher CCT may not result in much greater effectiveness of the lighting system for the circadian system. A much greater stimulation of the circadian system can be obtained if narrowband light sources peaking at short wavelengths can be used. It is important to remember, however, that light can also acutely impact biological rhythms, such as cortisol production and brain activities, via pathways other than melatonin suppression. Thus, short-wavelength light may not be the only, or even the most appropriate solution, depending on the application. Finally, it is important to always keep in mind that daylight from windows and clerestories is a circadian-effective light source, but, it should not be assumed that there will always be enough circadian stimulation from daylight in architectural spaces. Daylight levels in the room drop quickly as the distance from the window increases; 3 to 4 meters away from a window, daylight levels are quite low, even on a sunny day. It should be noted that if sunlight from the window penetrates the room, discomfort glare will cause occupants to draw blinds or shades, eliminating daylight entirely from the space.

During evening waking hours, the lighting system should provide no more than 50 lux at the cornea from a white light with low energy in the short-wavelength region of the spectrum. Relatively dim ambient evening light can be provided by light sources such as a $2700 \mathrm{~K}$ (warm-white) compact fluorescent lamp or LEDs. The dual lighting system provides people with a day/night circadian light ratio of about $24: 1$. The specifications for the proposed dual lighting system were determined from a mathematical model for human circadian phototransduction as it drives nocturnal melatonin suppression $^{556)}$. It should be emphasized, however, that the relationship between nocturnal melatonin suppression and circadian entrainment has yet to be clearly established. Nevertheless, the combination of "warm" light sources providing low light levels during the evening hours and "cool" light sources providing high light levels during the day promote circadian entrainment better than the static lighting systems currently designed.

The proposed, relatively dim evening lighting scheme is entirely consistent with current lighting recommendations and practice, and although the light levels recommended for daytime applications are relatively high compared to current practice, they are not difficult to achieve with current, high-efficacy fluorescent lighting systems, particularly if controls are incorporated into the design. If power density requirements are an issue, local supplementary lights from 470-nm LEDs can be integrated into the design. If re-designing the lighting scheme for one's entire living and workspace is not an option, individuals may opt to use 470-nm light goggles that deliver about 30 lux at the cornea for approximately one hour during the morning hours.

Light/dark patterns delivered to shift workers can 
be tuned to either entrain them to the new shift schedule or to simply promote acute alertness. It is recommended that whatever lighting system is used in healthcare applications, it does not promote suppression of melatonin during the nighttime hours. If individuals choose to expose themselves to circadian active light during the nighttime, it should be via personal lighting devices. Flexible lighting systems should be used in healthcare facilities because dayshift workers will need higher circadian stimulation during the day, while low circadian stimulation is recommended for the nightshift crew.

Of course, it is not reasonable to expect that individuals will remain in a single environment throughout the 24 hours, such as may occur with older adults living in nursing homes or assisted living facilities. Therefore, individual light treatment devices delivering tailored light/dark patterns to promote entrainment in various populations could be envisioned. These personal light treatment devices should be portable, lightweight, fashionable, and flexible enough to deliver and remove circadian light at appropriate times. In addition, such personal light treatment devices should be able to communicate with a Daysimeter (or a Dimesimeter) that collects light/dark exposure patterns to which a person is exposed. Moreover, the light treatment device should also communicate with a smartphone that interprets these light/dark patterns and is then able to recommend both current and future light exposure patterns to maintain circadian entrainment or to change the timing of the circadian clock. In the future, it is possible to envision the smartphone communicating with the building lighting systems itself so that proper lighting conditions for maintaining entrainment in an individual are achieved.

Even when designing light for optimal circadian entrainment, good visual conditions cannot be ignored. Lighting that provides high, glare-free illumination on the task with no direct or reflected view of the light source, soft shadows throughout the space, balanced illuminance levels, and good color rendering ${ }^{577}$ should be used.

Just as important as optimal task lighting, the proposed dual lighting scheme should include nightlights that reduce falls risk and help maintain sleep ${ }^{54) 58}$. In addition to impacting the aging circadian system, light can also impact the aging perceptual system. The perceptual system is comprised of a broad array of sensory information, such as sight, sound, smell, taste, touch, temperature, and pain that is interoperated by the central nervous system, via the process of sensation in one's environment. Sight and visibility are important for good perception; therefore, it is important to provide light that will promote stimulation of the perceptual system. Balance control is influenced by visual informa-

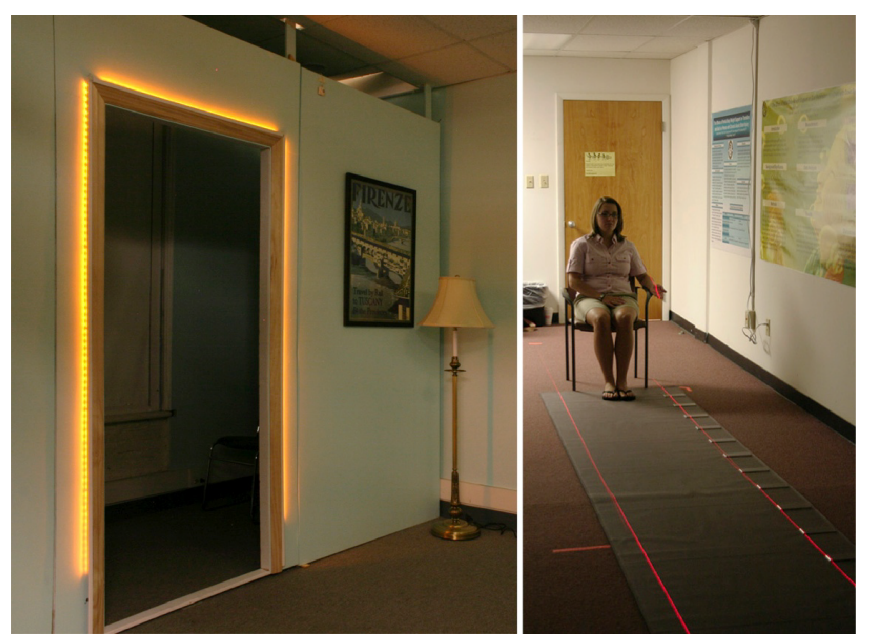

Figure 4 Novel nightlighting system delivering horizontal/vertical cues (left) ${ }^{58)}$ and horizontal lines outlining the pathway (right) ${ }^{60)}$.

tion as well as input from the vestibular and somatosensory systems. Any changes that occur to any of these systems and function will affect the other systems' capabilities of maintaining balance ${ }^{59)}$. In fact, studies have demonstrated that removal of visual cues by closing the eyes has been shown to result in increased body sway by $50 \%$. Recently, we showed that nightlights that provide horizontal and vertical perceptual cues were well accepted by caregivers and, more importantly, increased postural stability when older adults were transitioning from sitting to a standing position ${ }^{54 / 58)}$. Horizontal lines outlining the pathway on which subjects walked were compared to walking under night-lights alone (Figure 4$)^{60}$. The pathway lights increased gait velocity and reduced step length variability compared to the nightlights alone, particularly for those at greater risk of falling. Slower gait has been shown to be associated with higher risk for falls and has been correlated with lower scores on clinical balance scales ${ }^{61-63)}$. Increased gait variability, which quantifies the stride fluctuations during walking, has also been associated with increased risk for falls in less active older adults; therefore, lighting can be used to help minimize falls risks in older adults, including those at higher risk for falls.

The proposed nightlights should be controlled by motion sensors and provide dim illumination of the local environment (e.g., 1-2 lux at the cornea) as well as enhanced spatial perceptual information. As shown in Figure 4, the proposed nightlights should accent the rectilinear architectural features in the room, highlighting essential vertical and horizontal information people need to promote postural stability while navigating in an otherwise dim environment. However, the nightlights should emit light only when a person needs to 
move through the space at night. Because the proposed nightlight scheme would only turn on when motion is detected, occupants would not need to grope blindly for a light switch that, when found, would then activate a bright ambient lighting system.

\section{Conclusions}

The knowledge that light can impact a variety of biological functions in ways yet to be fully understood has led to new and exciting research. We now need to bring this knowledge into practice. The first step is to bring new light sources and lighting systems to the market, which will facilitate the implementation of this "second kind of light" into the built environment.

\section{Acknowledgements}

The author would like to acknowledge the Office of Naval Research, the US Green Buildings Council, the National Institute on Aging and the National Institute of Nursing Research for supporting the research presented in this review paper. The author would like to acknowledge Mark Rea, Barbara Plitnick, Brittany Wood, Anna Lok, Leora Radetsky, Andrew Bierman, Robert Hamner, Dennis Guyon, and Ines Martinovic of the Lighting Research Center for technical and editorial support.

\section{References}

(1) Berson, D. M., Dunn, F. A., Takao, M.: Phototransduction by retinal ganglion cells that set the circadian clock, Science, 295(5557), pp. 1070-1073 (2002).

(2) Rea, M. S., Figueiro, M. G. and Bullough, J. D.: Circadian photobiology: An emerging framework for lighting practice and research, Light Res. Tech., 34(3), pp. 177-190 (2002).

(3) Brainard, G. C., Hanifin, J. P., Greeson, J. M., Byrne, B., Glickman, G., Gerner, E. and Rollag, M. D.: Action spectrum for melatonin regulation in humans: Evidence for a novel circadian photoreceptor, J. Neurosci., 21(16), pp. 6405-6412 (2001).

(4) Thapan, K., Arendt, J. and Skene, D. J.: An action spectrum for melatonin suppression: Evidence for a novel non-rod, non-cone photoreceptor system in humans, J. Physiol., 535 (Pt 1), pp. 261-267 (2001).

(5) Rea, M. S., Figueiro, M. G., Bullough, J. D. and Bierman, A.: A model of phototransduction by the human circadian system, Brain Res. Rev., 50(2), pp. 213-228 (2005).

(6) Jewett, M., Rimmer, D., Duffy, J., Klerman, E., Kronauer, R. and Czeisler, C.: Human circadian pacemaker is sensitive to light throughout subjective day without evidence of transients, Am. J. Physiol., 273, pp. R1800-R1809 (1997).
(7) Khalsa, S. B., Jewett, M. E., Cajochen, C. and Czeisler, C. A.: A phase response curve to single bright light pulses in human subjects, J. Physiol., 549 (Pt 3), pp. 945-952 (2003).

(8) Glickman, G., Hanifin, J., Rollag, M., Wang, J., Cooper, H. and Brainard, G.: Inferior retinal light exposure is more effective than superior retinal exposure in suppressing melatonin in humans, J. Biol. Rhythms, 18(1), pp. 71-79 (2003).

(9) Hebert, M., Martin, S. K., Lee, C. and Eastman, C. I.: The effects of prior light history on the suppression of melatonin by light in humans, J. Pineal. Res., 33(4), pp. 198-203 (2002).

(10) Chang, A. M., Scheer, F. A. and Czeisler, C. A.: The human circadian system adapts to prior photic history, J. Physiol., 589 (Pt 5), pp. 1095-1102 (2011).

(11) Terman, J. S., Terman, M., Lo, E. S. and Cooper, T. B.: Circadian time of morning light administration and therapeutic response in winter depression, Arch. Gen. Psychiatry, 58(1), pp. 69-75 (2001).

(12) Figueiro, M. G. and Rea, M. S.: LEDs: Improving the sleep quality of older adults, CIE Midterm Meeting and International Lighting Congress, Leon, Spain (2005).

(13) Figueiro, M. G., Eggleston, G. and Rea, M. S.: Effects of Light Exposure on Behavior of Alzheimer's Patients-A Pilot Study, Light and Human Health: EPRI/LRO 5th International Lighting Research Symposium, pp. 151-156 (2002).

(14) Van Someren, E., Kessler, A., Mirmirann, M. and Swaab, D.: Indirect bright light improves circadian rest-activity rhythm disturbances in demented patients, Biol. Psychiatry, 41, pp. 955-963 (1997).

(15) Rivkees, S. A., Mayes, L., Jacobs, H. and Gross, I.: Rest-activity patterns of premature infants are regulated by cycled lighting, Pediatrics, 113(4), pp. 833-839 (2004).

(16) Auger, R. R., Burgess, H. J., Dierkhising, R. A., Sharma, R. G. and Slocumb, N. L.: Light exposure in adolescents with Delayed Sleep Phase Disorder, Sleep, 33 (Abstract Supplement), pp. A184-A185 (2010).

(17) Figueiro, M. G., Rea, M. S., Boyce, P. R., White, R. and Kolberg, K.: The effects of bright light on day and night shift nurses' performance and well-being in the NICU, Neonatal Intensive Care J. Perinatol Neonatol, 14(1), pp. 29-32 (2001).

(18) Stevens, R. G.: Electric power use and breast cancer: A hypothesis, Am. J. Epidemiol., 125, pp. 556-561 (1987).

(19) Fetveit, A., Skjerve, A. and Bjorvatn, B.: Bright light treatment improves sleep in institutionalized elderly-An open trial, Int. J. Geriatr. Psych., 18(6), pp. 520-526 (2003).

(20) Alessi, C. A., Martin, J. L., Webber, A. P., Cynthia, 
Kim E., Harker, J. O. and Josephson, K. R.: Randomized, controlled trial of a nonpharmacological intervention to improve abnormal sleep/wake patterns in nursing home residents, J. Am. Geriatr. Soc., 53(5), pp. 803-810 (2005).

(21) Murphy, P. J. and Campbell, S. S.: Enhanced performance in elderly subjects following bright light treatment of sleep maintenance in insomnia, J. Sleep Res., 5, pp. 165-172 (1996).

(22) Satlin, A., Volicer, L., Ross, V., Herz, L. and Campbell, S. S.: Bright light treatment for behavioral and sleep disturbances in patients with Alzheimer's disease, Am. J. Psychiatry, 149, pp. 1028-1032 (1992).

(23) Lyketosos, C., Lindell, Veiel L., Baker, A. and Steele, C.: A randomized, controlled trial of bright light therapy for agitated behaviors in dementia patients residing in long-term care, Int. J. Geriatr. Psychiatry, 14, pp. 520-525 (1999).

(24) Ancoli-Israel, S., Gehrman, P., Martin, J. L., Shochat, T., Marler, M., Corey-Bloom, J. and Levi, L.: Increased light exposure consolidates sleep and strengthens circadian rhythms in severe Alzheimer's disease patients, Behav. Sleep Med., 1(1), pp. 22-36 (2003).

(25) Yamadera, H., Takahashi, K. and Okawa, M.: A multicenter study of sleep-wake rhythm disorders: Therapeutic effects of vitamin B12, bright light therapy, chronotherapy and hypnotics, Psychiatry Clin. Neurosci., 50(4), pp. 203-209 (1996).

(26) Dowling, G. A., Burr, R. L., Van Someren, E. J., Hubbard, E. M., Luxenberg, J. S., Mastick, J. and Cooper, B. A.: Melatonin and bright-light treatment for rest-activity disruption in institutionalized patients with Alzheimer's disease, J. Am. Geriatr. Soc., 56(2), pp. 239-246 (2008).

(27) Sloane, P. D., Williams, C. S., Mitchell, C. M., Preisser, J. S ., Wood W., Barrick, A. L., Hickman, S. E., Gill, K. S., Connell, B. R., Edinger, J. et al.: Highintensity environmental light in dementia: Effect on sleep and activity, J. Am. Geriatr. Soc., 55(10), pp. 1524-1533 (2007).

(28) Royer, M., Ballentine, N. H., Eslinger, P. J., et al.: Light therapy for seniors in long term care, JAMA, 13(2), pp. 100-102 (2012).

(29) Figueiro, M. G., Saldo, E., Rea, M. S., Kubarek, K., Cunningham, J. and Rea, M. S.: Developing architectural lighting designs to improve sleep in older adults, Open. Sleep. J., 12, pp. 40-51 (2008).

(30) Riemersma-van der Lek, R. F., Swaab, D. F., Twisk, J., Hol, E. M., Hoogendijk, W. J. and Van Someren, E. J.: Effect of bright light and melatonin on cognitive and noncognitive function in elderly residents of group care facilities: A randomized controlled trial, JAMA, 299(22), pp. 2642-2655 (2008).

(31) Figueiro, M. G., Hamner, R., Higgins P., Hornick, T. and Rea, M. S.: Field measurements of light exposures and circadian disruption in two populations of older adults, J. Alzheimers Dis., 31(4), pp. 711-715 (2012).

(32) Gradisar, M., Dohnt, H. and Gardner, G., et al.: A randomized controlled trial of cognitive-behavior therapy plus bright light therapy for adolescent delayed sleep phase disorder, Sleep, 34(12), pp. 16711680 (2011).

(33) Crowley, S. J.. Acebo, C. and Carskadon, M. A.: Sleep, circadian rhythms, and delayed phase in adolescence, Sleep Med., 8 (2007), pp. 602-612 (2007).

(34) Carskadon, M. A., Labyak, S. E., Acebo, C. and Seifer, R.: Intrinsic circadian period of adolescent humans measured in conditions of forced desynchrony, Neurosci. Lett., 260(2), pp. 129-132 (1999).

(35) Aoki, H., Ozeki, Y. and Yamada, N.: Hypersensitivity of melatonin suppression in response to light in patients with Delayed Sleep Phase Syndrome, Chronobiol. Int., 18(2), pp. 263-271 (2001).

(36) Jenni, O. G., Achermann, P. and Carskadon, M. A.: Homeostatic sleep regulation in adolescents, Sleep, 28(11), pp. 1446-1454 (2005).

(37) Hagenauer, M. H., Perryman, J. I., Lee, T. M. and Carskadon, M. A.: Adolescent changes in the homeostatic and circadian regulation of sleep, Dev. Neurosci, 31(4), pp. 276-284 (2009).

(38) Figueiro, M. G. and Rea, M. S.: Lack of short-wavelength light during the school day delays dim light melatonin onset (DLMO) in middle school students, Neuroendocrinol. Lett., 31(1), p. 4 (2010).

(39) Figueiro, M. G. and Rea, M. S.: Evening daylight may cause adolescents to sleep less in spring than in winter, Chronobiol. Int., 27(6), pp. 1242-1258 (2010).

(40) Figueiro, M. G., Brons, J. A., Plitnick, B., Donlan, B. and Leslie, R. P.: Measuring circadian light and its impact on adolescents, Light Res. Tech., 43(2), pp. 201-215 (2011).

(41) Bierman, A., Klein, T. R. and Rea, M. S.: The Daysimeter: A device for measuring optical radiation as a stimulus for the human circadian system, Meas. Sci. Technol., 16, pp. 2292-2299 (2005).

(42) Badia, P., Myers, B., Boecker, M., Culpepper, J. and Harsh, J. R.: Bright light effects on body temperature, alertness, EEG and behavior, Physiol. Behav., 50(3), pp. 583-588 (1991).

(43) Cajochen, C., Zeitzer, J. M., Czeisler, C. A. and Dijk, D. J.: Dose-response relationship for light intensity and ocular and electroencephalographic correlates of human alertness, Behav. Brain Res., 115(1), pp. 75-83 (2000).

(44) Cajochen, C., Munch, M., Kobialka, S., Krauchi, K., Steiner, R., Oelhafen, P., Orgul, S. and Wirz-Justice, A.: High sensitivity of human melatonin, alertness, 
thermoregulation and heart rate to short wavelength light, J. Clin. Endo. Met., 90, pp. 1311-1316 (2005).

(45) Smith, M. R., Fogg, L. F. and Eastman, C. I.: A compromise circadian phase position for permanent night work improves mood, fatigue, and performance, Sleep, 32(11), pp. 1481-1489 (2009).

(46) Figueiro, M. G., Bullough, J. D., Bierman, A., Fay, C. R. and Rea, M. S.: On light as an alerting stimulus at night, Acta Neurobiol. Exp. (Wars), 67(2), pp. 171178 (2007).

(47) Figueiro, M. G., Bierman, A., Plitnick, B. and Rea, M. S.: Preliminary evidence that both blue and red light can induce alertness at night, BMC Neurosci., 10, pp. 105 (2009).

(48) Figueiro, M. G. and White, R. D.: Health consequences of shift work and implications for structural design, J. Perinatol., 33, pp. S17-S23 (2013).

(49) Phipps-Nelson, J., Redman, J. R., Dijk, D. J. and Rajaratnam, S. M.: Daytime exposure to bright light, as compared to dim light, decreases sleepiness and improves psychomotor vigilance performance, Sleep, 26(6), pp. 695-700 (2003).

(50) Vandewalle, G., Balteau, E., Phillips, C., Degueldre, C., Moreau, V., Sterpenich, V., Albouy, G., Darsaud, A., Desseilles, M., Dang-Vu, T. T. et al.: Daytime light exposure dynamically enhances brain responses, Curr. Biol., 16(16), pp. 1616-1621 (2006).

(51) Sahin, L. and Figueiro, M. G.: Alerting effects of short-wavelength (blue) and long-wavelength (red) lights in the afternoon, Physiol. Behav., 116-117C, pp. 1-7 (2013).

(52) Blask, D., Sauer, L., Dauchy, R., Holowachuk, E., Ruhoff, M. and Kopff, H.: Melatonin inhibition of cancer growth in vivo involves suppression of tumor fatty acid metabolism via melatonin receptormediated signal transduction events, Cancer Res., 59, pp. 4793-4701 (1999).

(53) Schernhammer, E., Laden, F., Speizer, F., Willett, W., Hunter, D. and Kawachi, I.: Rotating night shifts and risk of breast cancer in women participating in the Nurses' Health Study, J. Natl. Cancer Inst., 93, pp. 1563-1568 (2001).

(54) Figueiro, M. G.: A proposed 24 h lighting scheme for older adults, Light Res. Tech., 40(2), pp. 153-160 (2008).

(55) Hanford, N. and Figueiro, M. G.: Light therapy and Alzheimer's disease and related dementia: Past, present, and future, J. Alzheimers Dis., 33(4), pp. 913-922 (2013).

(56) Rea, M. S ., Figueiro, M. G., Bierman, A. and Hamner, R.: Modeling the spectral sensitivity of the human circadian system, Light Res. Tech., 44(4), pp. 386-396 (2012).

(57) Rea, M. S. and Freyssinier-Nova, J. P.: Color rendering: A tale of two metrics, Color Res. Appl., 33(3), pp. 192-202 (2008).

(58) Figueiro, M. G., Gras, L. Z. and Rea, M. S. and Plitnick B.: Lighting for improving balance in older adults with and without risk for falls, Age Ageing, 41(3), pp. 392-395 (2012).

(59) Paulus, W. M., Straube, A. and Brandt, T.: Visual postural performance after loss of somatosensory and vestibular function, J. Neurol. Neurosurg. Psych., 50, pp. 1542-1545 (1987).

(60) Figueiro, M. G., Plitnick, B., Rea, M. S., Gras, L. Z. and Rea, M. S.: Lighting and perceptual cues: Effects on gait measures of older adults at high and low risk for falls, BMC Geriatrics, 11-49 (2011).

(61) Verghese, J., Holtzer, R., Lipton, R. B. and Wang, C.: Quantitative gait markers and incident fall risk in older adults, J. Gerontol A. Biol. Sci. Med. Sci., 64(8), pp. 896-901 (2009).

(62) Callisaya, M. L., Blizzard, L., Schmidt, M. D., McGinley, J. L. and Srikanth, V. K.: Ageing and gait variability - A population-based study of older people, Age Ageing, 39(2), pp. 191-197 (2010).

(63) Shumway-Cook, A., Brauer, S. and Woollacott, M.: Predicting the probability for falls in communitydwelling older adults using the Timed Up \& Go Test, Phys. Ther., 80(9), pp. 896-903 (2000). 